\title{
Load sharing in lumbar spinal segment as a function of location of center of rotation
}

\author{
Laboratory investigation
}

\author{
Yunus Alapan, M.Sc., ${ }^{1}$ Semih Sezer, Ph.D., ${ }^{1}$ Cihan Demir, Ph.D., ${ }^{1}$ Tuncay Kaner, M.D., ${ }^{2}$ \\ and Serkan İnCeoĞLu, Ph.D. ${ }^{3}$ \\ ${ }^{1}$ Department of Mechanical Engineering, Yildiz Technical University; ${ }^{2}$ Department of Neurosurgery, School \\ of Medicine, Istanbul Medeniyet University, Istanbul, Turkey; and ${ }^{3}$ Department of Orthopedic Surgery, Loma \\ Linda University, Loma Linda, California
}

\begin{abstract}
Object. The center (axis) of rotation (COR) in the lumbar spine has been studied well. However, there is limited information on the kinetic and kinematic consequences of imposed shift in the location of the COR, although this type of shift can be seen after surgeries using motion preservation or dynamic stabilization devices. The objective of this study was to assess the kinetic and kinematic changes in the lumbar spinal segment due to various imposed CORs.

Methods. A 3D finite element model of the L4-5 segment was constructed and validated. The segment was loaded under a 7.5-Nm bending moment while constrained to rotate about various imposed CORs in the sagittal and axial motion planes. Range of motion, ligament forces, facet loads, and disc stresses were measured.

Results. The present model showed an agreement with previous in vitro and finite element studies under the same load and boundary conditions. Range of motion, facet forces, disc stresses, and ligament loads showed a strong association with the location of the COR.

Conclusions. Acute alterations in the location of the COR can significantly change the load sharing characteristics within the spine segment. The normal location of the COR is a result of the tendency of the vertebra to move in the path of least cumulative resistance.

(http://thejns.org/doi/abs/10.3171/2014.1.SPINE13426)
\end{abstract}

\section{Key Words • lumbar spine • center of rotation • finite element • load sharing - motion preservation}

$\mathrm{T}$ HE kinematics of the spine is expressed as how much the segment moves (range of motion [ROM]) and how it moves (center of rotation [COR] or axis of rotation). The kinetic consequences of spinal motion are generally expressed as facet pressures, disc stresses, and ligament forces. The normal motion of the healthy spine is governed by a delicate balance and interaction of these kinetic and kinematic factors. A change in this orchestrated motion can cause further complications in the spine as seen in adjacent-level diseases developing after fusion surgeries.

The progression of disc degeneration in the adjacent levels after immobilizing a segment of the spine has steered researchers' interest to motion preservation or dy-

Abbreviations used in this paper: ALL = anterior longitudinal ligament; $\mathrm{CL}=$ capsular ligament; $\mathrm{COR}=$ center of rotation; $\mathrm{FE}=$ finite element; ISL = interspinous ligament; ITL = intertransverse ligament; $\mathrm{LF}=$ ligamentum flavum; PLL = posterior longitudinal ligament; $\mathrm{ROM}=$ range of motion; $\mathrm{SSL}=$ supraspinous ligament . namic stabilization strategies and thus has accelerated efforts for more detailed studies on spinal kinematics. The concept of instantaneous COR in spinal motion has consequently gained a great deal of interest and practical significance in the clinical setting. Many studies have described the COR or helical axis of normal spinal motion., ${ }^{4,22,22}$, 29,32,35,39,44,46,49 Researchers have also evaluated the biomechanical performance of various artificial disc implants and posterior dynamic stabilization devices in mimicking the normal motion of the spine by using these qualitative measures of spinal motion. ${ }^{3,4,18,19,22,29,43,46}$ While both the COR and the helical axis are useful in describing the relative motion of one vertebra with respect to another in 2 dimensions and 3 dimensions, respectively, the former has gained popularity because it is easy to calculate and apply on plain radiography, and thus it is understood by nonexperts.

This article contains some figures that are displayed in color online but in black-and-white in the print edition. 


\section{Load sharing with respect to COR location}

Researchers have shown that the COR of a vertebra in the normal spine is located in the posterior one-third of the disc in sagittal motion. , $^{3,29,45,49}$ It has also been shown that structural changes in the spinal segment such as disc degeneration or any kind of column instability can cause a shift in the COR. ${ }^{1,2,12,14,25,38,39,44}$ However, there is little or no information on how an acute shift in the COR affects the kinetics and kinematics of the lumbar spine. Indeed, such knowledge could enhance our understanding of the consequences of poorly designed or incorrectly placed motion preservation devices. Moreover, the contributions of the structural elements of the spinal segment to the formation of the normal COR could be better understood. Therefore, in this study, we attempted to describe the changes in the load-sharing characteristics in the lumbar spine segment in response to a series of imposed COR points using a computational model.

\section{Methods}

A 3D, nonlinear finite element (FE) model of the L4-5 segment was generated using the CT images (slice thickness $1 \mathrm{~mm}$ ) obtained from the Visible Human Project of the United States National Library of Medicine. ${ }^{41}$ Following the 3D reconstruction of the model from CT images using ImageVis3D (http://www.sci.utah.edu/software/imagevis3d.html), CATIA (Dassault Systems) software was used to convert the reconstruction to a solid model. Finite element modeling of the solid model was conducted in ANSA (BETA CAE Systems SA) and then imported into ANSYS (Swanson Analysis) for nonlinear FE analysis. The FE model consisted of 1230 link, 300 spring, and 270,324 solid elements (Fig. 1).

The L-4 and L-5 vertebrae consisted of cortical bone, trabecular bone, and posterior bony elements. Cortical bone was modeled using wedge solid elements with 0.5 mm thickness. ${ }^{7}$ Trabecular bone and posterior bony elements were formed with pyramid and tetrahedral elements.

The intervertebral disc was composed of nucleus pulposus, annulus fibrosus, and cartilaginous endplates at the inferior and superior ends. The nucleus pulposus was positioned slightly posterior to the disc center and filled $43 \%$ of the total disc volume. ${ }^{40}$ Furthermore, the annulus was modeled with annulus fibers and annulus ground substance. The orientations of the annulus fibers increased continuously from $\pm 23^{\circ}$ at the anterior side to $\pm 58^{\circ}$ at the posterior side, and the ratio of fiber volume to the surrounding annulus ground substance volume changed from $5 \%$ to $23 \%$ from the inner layers through the outer layers. ${ }^{21}$ The annulus ground substance, nucleus, and cartilaginous endplates were modeled with hexahedral solid elements, and the annulus fibers were modeled with tension-only link elements.

Facet joints were simulated by defining a frictionless contact between facet cartilage layers with a contact stiffness of $200 \mathrm{~N} / \mathrm{mm}^{36}$ along with a $0.5-\mathrm{mm}$ initial gap between facet cartilage layers. ${ }^{28}$ Facet cartilage layers were modeled with hexahedral solid elements.

The material properties of the cortical bone, trabecular bone, and posterior bony elements ${ }^{5,34,47}$ as well as facet cartilage layers, ${ }^{20}$ nucleus, annulus ground substance, cartilaginous endplates, annulus fibers, ${ }^{7,8,33}$ and ligaments ${ }^{24,26}$, $30,33,47$ were adapted from the literature (Table 1). Because of the large distortions in some of the disc elements during rotation about Points 4 and 5 in extension, analysis could only be completed when the elasticity modulus of the nucleus was doubled. Such adjustment in the material property of the nucleus was deemed acceptable because it caused less than a $2 \%$ difference in all calculations. Similar large deformation problems and solutions were reported in experimental studies regarding the imposed COR. ${ }^{9,23}$

\section{Load and Boundary Conditions}

Validation. The nodes of the inferior endplate of the L-5 vertebra were fixed, while the L-4 vertebra was allowed to move in all directions. A pure bending moment of $7.5 \mathrm{Nm}$ was applied in 3 anatomical planes by using rigid beam elements, which were concentrated in a single node at one end and attached to the superior endplate of L-4 at the other end. This technique allowed us to apply homogeneously distributed loads to the endplate.

COR Simulations. Pure rotations around predetermined CORs were simulated by applying 7.5-Nm bending moments in sagittal and axial planes. The loads were ap-
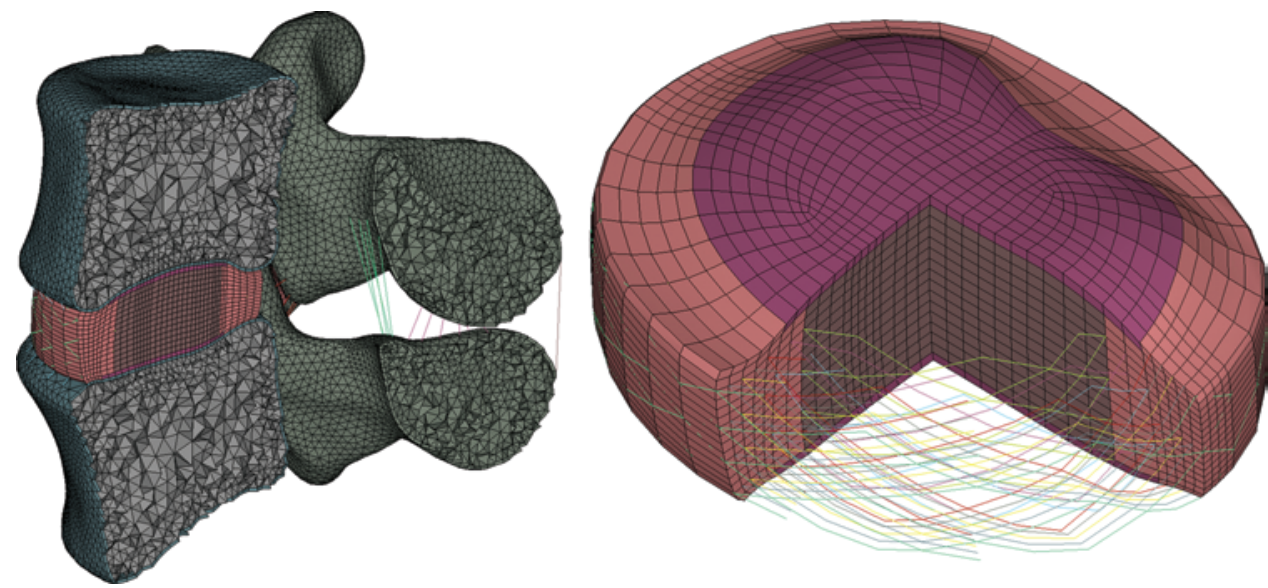

FIG. 1. A section of the L4-5 finite element model (left) and intervertebral disc with annulus fibers exposed (right). 
TABLE 1: Material property definitions used in the finite element model*

\begin{tabular}{|c|c|c|c|c|c|}
\hline \multirow[b]{2}{*}{ Structure } & \multirow[b]{2}{*}{ Young's Modulus (MPa) } & \multirow[b]{2}{*}{ Poisson's Ratio } & \multirow[b]{2}{*}{ Cross-Section $\left(\mathrm{mm}^{2}\right)$} & \multicolumn{2}{|c|}{ Mooney-Rivlin } \\
\hline & & & & $\mathrm{C}_{1}$ & $\mathrm{C}_{2}$ \\
\hline \multicolumn{6}{|l|}{ vertebra } \\
\hline cortical bone & 5000 & 0.3 & & & \\
\hline cancellous bone & 50 & 0.2 & & & \\
\hline posterior elements & 3000 & 0.3 & & & \\
\hline facet cartilage layers & 10.4 & 0.4 & & & \\
\hline \multicolumn{6}{|l|}{ intervertebral disc } \\
\hline nucleus & & & & 0.017 & 0.004 \\
\hline nucleus $†$ & & & & 0.034 & 0.008 \\
\hline annulus fibers & 175 & 0.3 & $0.1-0.78$ & & \\
\hline annulus ground substance & & & & 0.18 & 0.045 \\
\hline cartilage endplate & 23.8 & 0.4 & & & \\
\hline
\end{tabular}

plied through rigid beam elements with one end concentrated in a single node, which was the designated COR, and the other end attached to the superior endplate of the L-4 vertebra. The node, on which the loads were applied, was constrained in all translational directions to obtain a fixed COR scenario. The nodes of the inferior endplate of L-5 were constrained in all directions.

For predetermined COR locations in sagittal bending, 5 points were selected on the intersection line of the sagittal and axial midplanes of the disc (Fig. 2). To investigate a case of vertical shift in the COR, additional points (6F [flexion] and $6 \mathrm{E}$ [extension]) were selected in the sagittal midplane, that is, the same plane as the aforementioned 5 points, and one disc height below Point 4 for flexion and Point 3 for extension. Points 3 and 4 were identified as the locations with the highest ROM, which indicated the proximity of the natural CORs of the segment in flexion and extension. For axial rotation, 4 points were selected on the intersection line of the middisc axial plane and in a plane that passed through the center of the disc and left facet (Fig. 2). Lateral bending motion was not studied to keep the study concise.

\section{Data Analysis}

For 6 different COR points in the sagittal plane and 4 different COR points in the axial plane, intersegmental

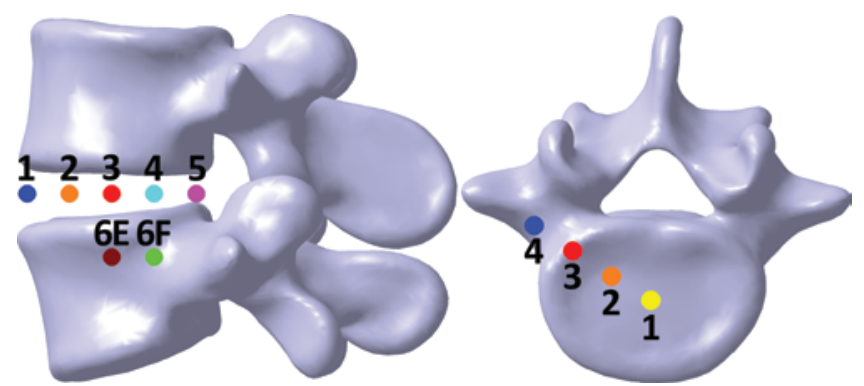

FIG. 2. Designated COR points in the sagittal (left) and axial (right) planes.
ROM, facet forces, ligament loads, and maximum annulus stresses were calculated. Ligament loads were evaluated as a summation of forces on elements of each ligament, and facet force was calculated as a vector summation of 3D components (x, y, and $\mathrm{z}$ ) of each facet (left and right). Maximum annulus stress was defined as maximum Von Mises stress obtained during motion. For ease of comparison, all results at each COR point were normalized to that of Points 3 and 4 in extension and flexion, respectively.

\section{Results}

\section{Validation}

The present FE model of the L4-5 segment showed agreement in ROM with previous FE and in vitro studies in the literature having the same boundary and load conditions (Fig. 3). ${ }^{13,16,19,31,37,48}$

\section{Effects of COR Alteration}

Range of Motion. In flexion, the minimum and maximum ROM values were obtained at Points 1 and 4, respectively (Table 2 and Fig. 4). In extension, the ROM values were minimum at Point 1 and maximum at Point 3. Change in ROM due to vertical shift was higher in flexion $(5 \%)$ than in extension $(2 \%)$. In left axial rotation, the ROM obtained at Points $1-4$ were $1.44^{\circ}, 1.75^{\circ}, 1.92^{\circ}$, and $1.78^{\circ}$, respectively.

Maximum Annulus Stress. In flexion, maximum annulus stress had a steady trend initially and reached a maximum during rotation around Point 5, which was similar to that of Point 6F (Fig. 5). Similarly, in extension, maximum annulus stress in Points 4-6 was higher than in Points 1-3.(Fig. 6).

Facet Loads. There were no facet forces generated in flexion during bending about Points $1-5$ while $5.65 \mathrm{~N}$ was recorded when the COR was shifted inferiorly. In extension, the average facet force was maximum at Point 3 (Ta- 


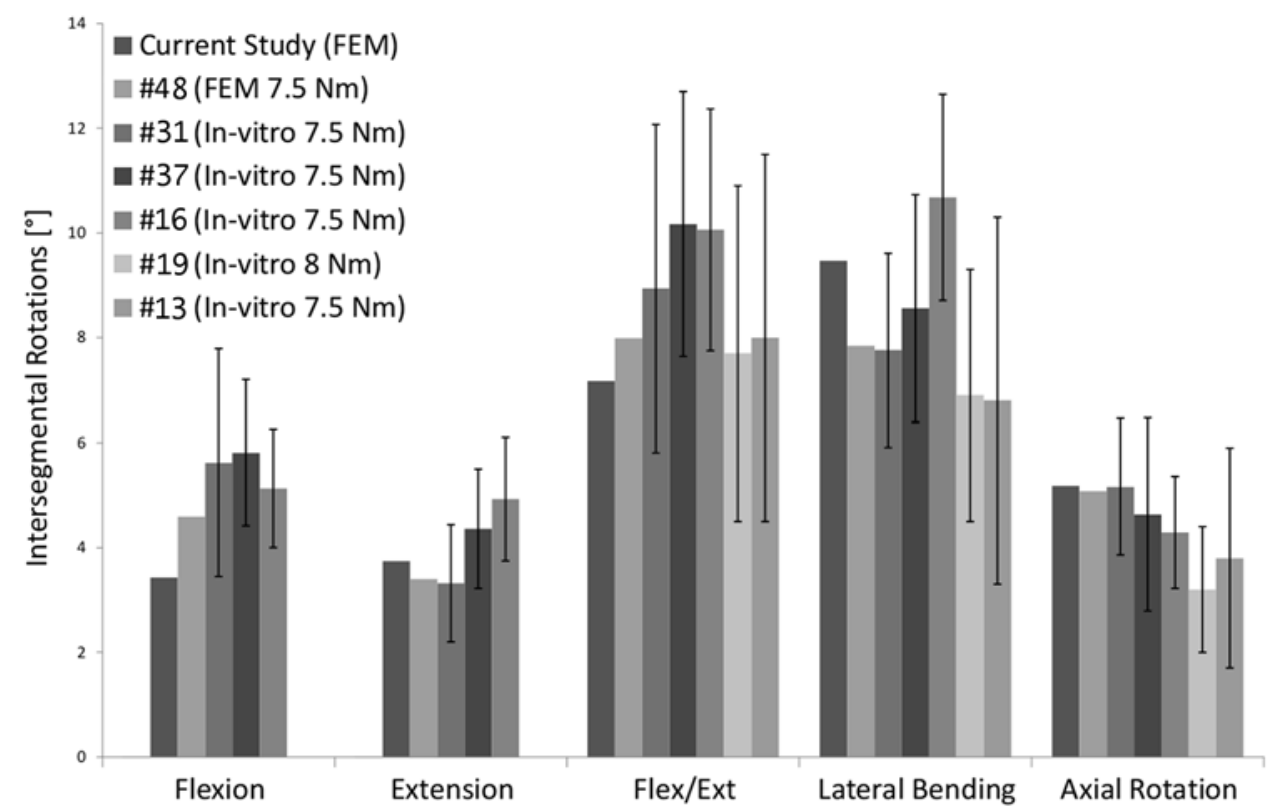

FIG. 3. Intersegmental ROM data from the current study compared with previous FE models (FEM) and in vitro experiment studies in the literature. Flex/Ext = flexion/extension; \# = reference number of the study. The bars represent the mean and the whiskers the SD.

ble 2 and Fig. 6). The average facet force obtained during motion around Point $6 \mathrm{E}$ was less than that of Point 3. In left axial rotation, there were no facet forces at the left facet joint. The facet force at the right facet joint was the highest for rotation about Points 1 and 2 (Table 2 and Fig. 7).

Ligament Loads. In flexion, all ligament loads increased through the initial anterior COR locations and then decreased toward the posterior COR locations (Fig. 5). The supraspinous ligament (SSL) was not activated when the COR was at Points 1 and 2, but reached a maximum at Point 4 . The anterior longitudinal ligament (ALL) was barely activated during flexion in all COR points except for Point 1 . When the COR was moved to Point $6 \mathrm{~F}$ in flexion, all ligament loads increased again.

In extension, only the ALL and capsular ligament (CL) experienced loading while the other ligaments remained passive. The ALL showed continuously increasing load when the COR moved from Point 1 through 4, then decreased at Point 5. The CL had a maximum load when the COR was at Point 3. During the rotation about Point 6E in extension, the load of the ALL decreased and that of the CL increased compared with those obtained at Point 3 (Fig. 6).

In left axial rotation, there was no load on the SSL, and the loads on the posterior longitudinal ligament (PLL), interspinous ligament (ISL), and ligamentum flavum (LF) were negligible compared with others. When the COR moved from Point 1 through 4, the intertransverse ligament (ITL) and right CL loads decreased continuously, the left CL load remained unchanged, and the ALL load had a minimum at Point 2 and increased thereafter (Fig. 7).

\section{Discussion}

The current study investigated the relationship be- tween various imposed COR locations and ROM, facet joint forces, maximum annulus stress, and ligament loads. The results showed that COR alterations in the sagittal and axial planes within the span of the disc space led to changes in the kinematics and load sharing characteristics of the segment.

The simulation results showed that, in the sagittal plane, an anteriorly positioned COR yielded the lowest ROM while the highest ROM was obtained when the COR was within the central and posterior disc. The pattern was in agreement with an experimental study by Panjabi et al., ${ }^{23}$ where the researchers applied a sagittal bending load to a lumbar segment with imposed CORs. They found that the largest ROM was around the center or posterior wall of the vertebra. These results were intuitive because the natural COR of a healthy lumbar segment was shown to be around the posterior one-third of the disc. ${ }^{3,4,29,45,49}$ Similarly, in the current study, the axial rotation behavior of the segment indicated that the largest motion occurred around the natural COR of the segment. , $^{12,32}$

The change in the COR location varied the path of the motion and thus the stress distribution within the disc, the stretch of each ligament, and the moment arm, that is, the distance to the COR, of each ligament. Hence, at any COR location, the rotation of the vertebra caused a new composition of load distribution among the elements of the segment. The change in ROM at each COR point observed in the current results can be explained by this variation in the load sharing characteristics.

In our study we used a flexibility protocol because it is the standard method in spine testing; that is, we applied a known load to the segment and observed the resulting motion. To provide further insight into the kinematics of load sharing within the spinal segment during a change in COR location, we conducted a flexion test with stiffness protocol, where $0.96^{\circ}$ bending was applied, which was the 
TABLE 2: Range of motion, maximum annulus stress, facet contact forces, and ligament loads with respect to imposed COR(s)

\begin{tabular}{|c|c|c|c|c|c|c|}
\hline Parameter & Point 1 & Point 2 & Point 3 & Point 4 & Point 5 & Point $6^{*}$ \\
\hline \multicolumn{7}{|l|}{ flexion } \\
\hline $\operatorname{ROM}\left({ }^{\circ}\right)$ & 0.96 & 1.61 & 2.63 & 3.39 & 2.60 & 3.24 \\
\hline max annulus stress (MPa) & 0.54 & 0.61 & 0.54 & 0.65 & 0.80 & 0.79 \\
\hline contact force $(\mathrm{N})$ & & & & & & 11.31 \\
\hline ITL (N) & 24.4 & 32.3 & 39.05 & 33.18 & 13.47 & 30.13 \\
\hline PLL (N) & 1.55 & 3.58 & 6.86 & 8.43 & 6.34 & 8.00 \\
\hline ALL (N) & 13.5 & 1.03 & 0.01 & 1.42 & 3.08 & 1.77 \\
\hline SSL (N) & 0.00 & 0.00 & 3.79 & 6.16 & 0.91 & 5.40 \\
\hline ISL (N) & 0.11 & 11.87 & 35.38 & 44.50 & 9.01 & 35.14 \\
\hline LF (N) & 8.44 & 11.41 & 14.22 & 13.57 & 7.95 & 14.16 \\
\hline$C L(N)$ & 36.7 & 46.61 & 55.06 & 52.08 & 31.9 & 47.01 \\
\hline \multicolumn{7}{|l|}{ extension } \\
\hline $\mathrm{ROM}\left({ }^{\circ}\right)$ & 1.33 & 2.19 & 3.32 & 3.11 & 1.90 & 3.38 \\
\hline max annulus stress (MPa) & 0.63 & 0.71 & 0.65 & 0.87 & 0.74 & 0.81 \\
\hline contact force $(\mathrm{N})$ & 93.8 & 150 & 195.5 & 107.9 & 23.8 & 155.7 \\
\hline ITL (N) & 0.00 & 0.00 & 0.00 & 0.00 & 1.85 & 0.00 \\
\hline PLL (N) & 1.34 & 1.40 & 0.84 & 0.00 & 0.00 & 0.92 \\
\hline ALL $(N)$ & 39.6 & 168 & 931.9 & 1997 & 1532 & 906.7 \\
\hline SSL (N) & 0.00 & 0.00 & 0.00 & 0.00 & 0.00 & 0.00 \\
\hline ISL (N) & 0.00 & 0.00 & 0.00 & 0.00 & 0.00 & 0.00 \\
\hline $\operatorname{LF}(\mathrm{N})$ & 0.00 & 0.00 & 0.00 & 0.00 & 0.00 & 0.00 \\
\hline $\mathrm{CL}(\mathrm{N})$ & 5.18 & 11.98 & 17.77 & 8.53 & 0.38 & 24.40 \\
\hline \multicolumn{7}{|l|}{ axial rotation } \\
\hline $\operatorname{ROM}\left({ }^{\circ}\right)$ & 1.44 & 1.75 & 1.92 & 1.78 & & \\
\hline max annulus stress (MPa) & 0.30 & 0.36 & 0.43 & 0.51 & & \\
\hline contact force $(\mathrm{N})$ & 152.5 & 155.6 & 120.4 & 41.44 & & \\
\hline ITL (N) & 12.97 & 7.15 & 1.11 & 0.00 & & \\
\hline PLL (N) & 0.18 & 0.35 & 0.49 & 0.53 & & \\
\hline ALL (N) & 12.13 & 5.22 & 30.5 & 105.9 & & \\
\hline SSL (N) & 0.00 & 0.00 & 0.00 & 0.00 & & \\
\hline ISL (N) & 1.02 & 1.79 & 0.27 & 0.00 & & \\
\hline LF (N) & 3.14 & 2.23 & 0.32 & 0.00 & & \\
\hline It $C L(N)$ & 22.4 & 25.94 & 27.33 & 23.68 & & \\
\hline rt CL (N) & 5.38 & 3.37 & 1.65 & 1.35 & & \\
\hline
\end{tabular}

* Point 6 comprises Point $6 \mathrm{~F}$ for flexion and Point $6 \mathrm{E}$ for extension.

ROM value obtained at Point 1 . It was observed that for the same amount of flexion, the ratio of ligament loads generated during rotation about Point 1 to those at Point 3 was 2 for the ITL, 8 for the PLL, and 1 for the FL and $\mathrm{CL}$, while the ratios of the annulus, annulus fibers, and nucleus stresses were 3, 2, and 3, respectively. Moreover, in the same comparison, the ratio of moments generated by the ligaments was 3 for the ITL, 16 for the PLL, and 2 for the FL and the CL because of the change in the moment arm lengths. Furthermore, comparison of the reaction moments showed that only $2 \mathrm{Nm}$ was required for $0.96^{\circ}$ flexion about Point 3 while $7.5 \mathrm{Nm}$ was required for the same amount of flexion about Point 1 . This inverse approach to the COR phenomenon showed that the COR was a result of the tendency of the vertebra to move in the path of least cumulative resistance.

The reduction in the ROM in sagittal motion was strikingly high and indicated that suboptimally designed and/or placed motion preservation devices can theoretically prevent motion. Dooris et al. showed that an 8-mm anterior shift in the placement of a ball-and-socket-type artificial disc device caused a 19\% reduction in flexion compared with its proper posterior position, which is in agreement with current findings. ${ }^{6}$ Supporting these observations, Rohlmann et al. showed an almost $25 \%$ reduction in flexion when an artificial disc device was placed $2 \mathrm{~mm}$ anterior to its optimal position. ${ }^{27}$ Although in our study we did not simulate any axial loading as done in these 


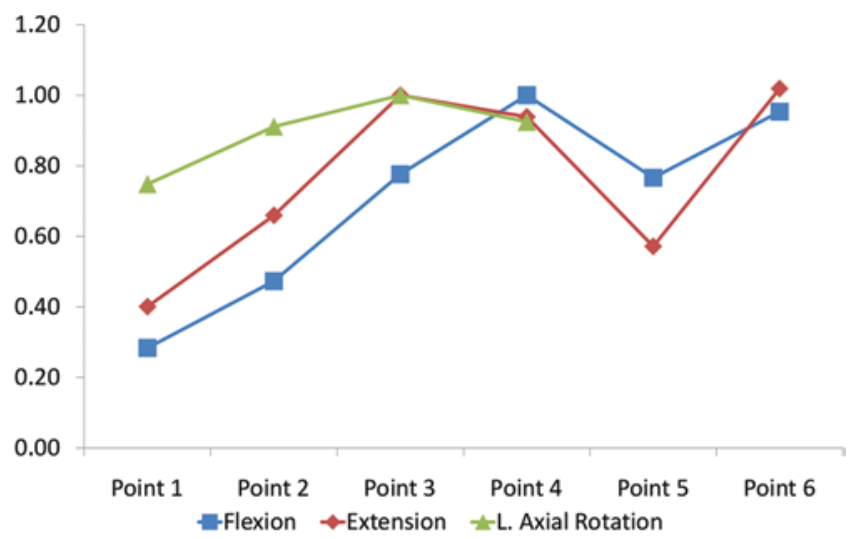

Fig. 4. Range of motion with respect to imposed $\operatorname{COR}(\mathrm{s})$ as normalized to those at either Point 3 or 4 , in flexion, extension, and left axial rotation.

aforementioned studies, our results can explain some of those changes reported in segmental kinetics and kinematics. It is also important to note that some ligaments as well as the disc and the facets experienced increased stress at the shifted CORs compared with the relatively natural COR. Prolonged exposure to increased stress can deteriorate the integrity of these structures and accelerate the degeneration process.

Posterior dynamic stabilization devices, unlike balland-socket disc replacements, do not impose a fixed COR on the segment. However, due to the nature of implant designs, variability in surgical technique, and patient anatomy, the resulting motion may not necessarily coincide with the natural COR of the segment. In a study by Niosi et al., it was shown that a lumbar construct with a posterior dynamic stabilization device exhibited a COR located in the spinal canal in sagittal motion. ${ }^{22}$ The specimens with the COR shifted posteriorly had a decreased ROM in flexion and extension as observed in the current study. Posterior dynamic stabilization leaves the disc untouched and provides load sharing by restricting motion and transferring the load posteriorly. The long-term complications of posterior dynamic systems have been reported as adjacent-segment degeneration, foraminal narrowing, and flat-back syndrome due to fixation loosening or

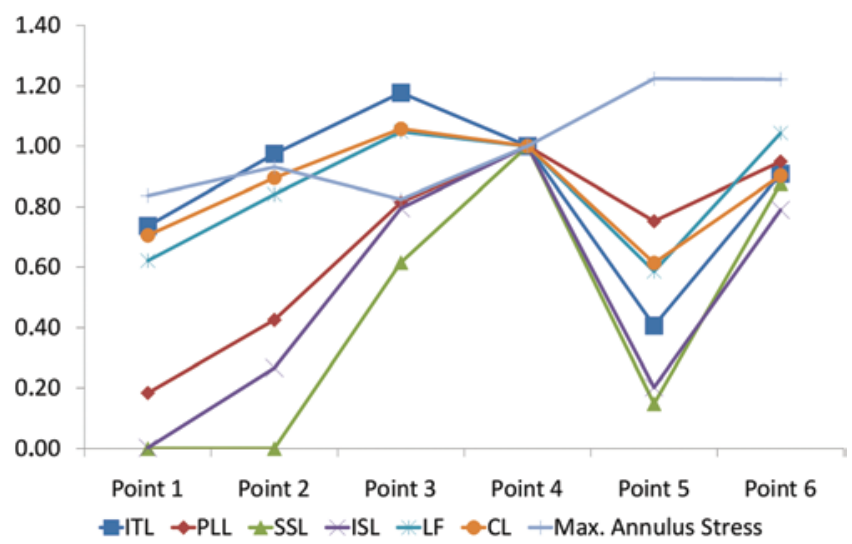

FIG. 5. Ligament loads and maximum annulus stress with respect to imposed COR(s) as normalized to those at Point 4, in flexion.

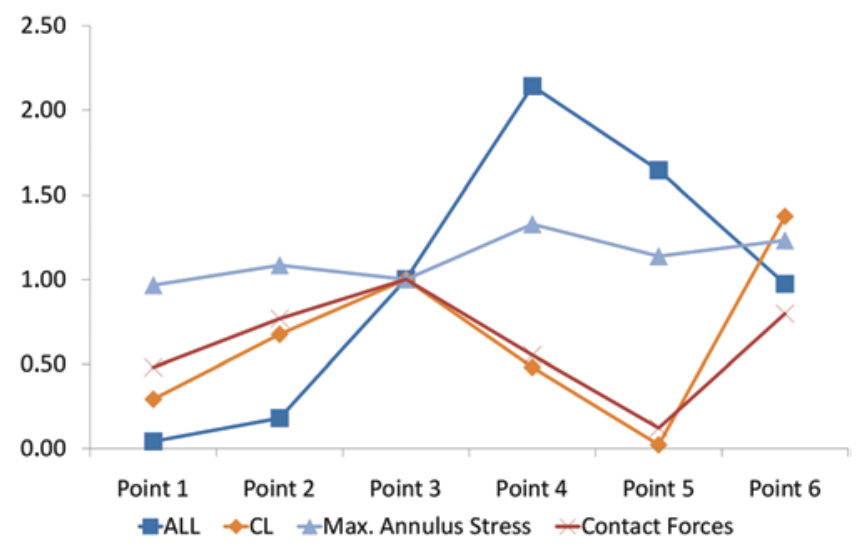

Fig. 6. Ligament loads, facet contact forces, and maximum annulus stress with respect to imposed $\mathrm{COR}(\mathrm{s})$ as normalized to those at Point 3 , in extension.

breakage. ${ }^{10,11,15,17,42}$ These complications could be related to the altered kinematics as explained above.

A shift in the location of the COR can also occur as a result of structural deterioration, such as disc degeneration or any kind of column deficiency. ${ }^{12,14,25,38,39}$ In a mathematical modeling study, Bogduk et al. showed that possible pathological conditions, such as muscle spasm or ligament weakness, can change the location of the COR. ${ }^{1}$

The computational model used in the present study shares certain limitations as other models in the literature on the simulation of spinal motion. We used a spine model developed from previously obtained CT images of a single L4-5 motion segment and thus did not have a physical specimen for cadaveric testing. We validated the reliability of our model by comparing our results with previous experimental and computational data reported for the same segmental spinal motion. Hence, the results of our subject-specific model cannot be an absolute reference for the general population. Instead, the trends and relative changes in the observations should be carefully considered as the useful message. Rather than studying the $3 \mathrm{D}$ motion (that is, the axis of rotation), we studied the motion of the spinal segment-simulated loading conditions in the principal anatomical directions and reported

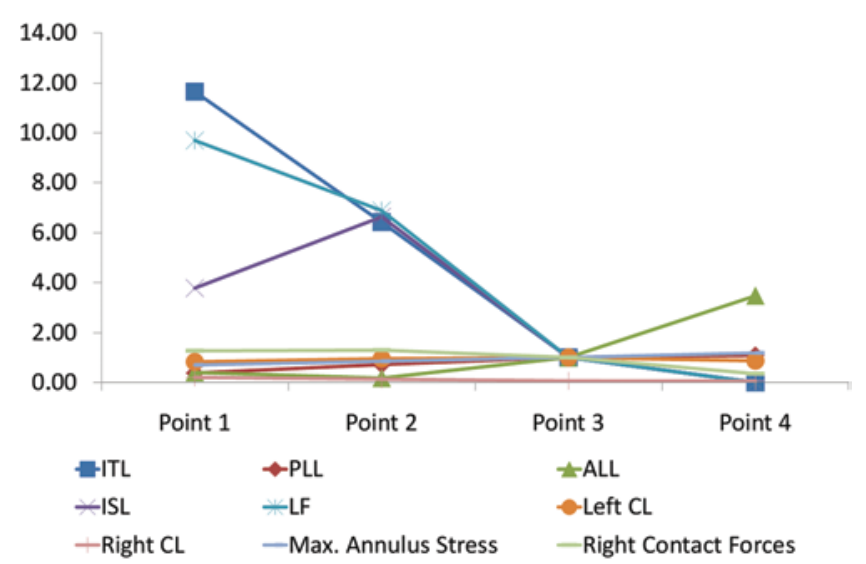

Fig. 7. Ligament loads, facet contact forces, and maximum annulus stress with respect to imposed $\mathrm{COR}(\mathrm{s})$ as normalized to those at Point 3 , in left axial rotation. 
the "center of rotation." The planar analysis is easier to report and understand and allowed us to compare our results with the widely available in vitro published data.

Our model does not include muscles to maintain similarities with published experimental in vitro data. More elaborate future models might include the muscles and other spinal levels. Moreover, parametric models can also be formulated to account for the effect of variability in parameters, such as geometries and loading.

\section{Conclusions}

This study comprehensively investigated the changes in ROM, ligament loads, annulus stresses, and facet forces with respect to imposed COR locations in the lower lumbar spine. This comparative analysis suggested that the load sharing characteristics of the spine were strongly correlated with the location of the COR and that the location of the COR was a result of the tendency of the vertebra to move in the path of least cumulative resistance.

\section{Disclosure}

The authors report no conflict of interest concerning the materials or methods used in this study or the findings specified in this paper.

Author contributions to the study and manuscript preparation include the following. Conception and design: all authors. Acquisition of data: İnceoğlu, Alapan. Analysis and interpretation of data: all authors. Drafting the article: all authors. Critically revising the article: all authors. Reviewed submitted version of manuscript: all authors. Approved the final version of the manuscript on behalf of all authors: İnceoğlu. Administrative/technical/material support: Demir, Sezer. Study supervision: İnceoğlu.

\section{References}

1. Bogduk N, Amevo B, Pearcy M: A biological basis for instantaneous centres of rotation of the vertebral column. Proc Inst Mech Eng H 209: 177-183, 1995

2. Cossette JW, Farfan HF, Robertson GH, Wells RV: The instantaneous center of rotation of the third lumbar intervertebral joint. J Biomech 4:149-153, 1971

3. Cunningham BW, Gordon JD, Dmitriev AE, Hu N, McAfee PC: Biomechanical evaluation of total disc replacement arthroplasty: an in vitro human cadaveric model. Spine (Phila Pa 1976) 28:S110-S117, 2003

4. Cunningham BW, Hu N, Zorn CM, McAfee PC: Biomechanical comparison of single- and two-level cervical arthroplasty versus arthrodesis: effect on adjacent-level spinal kinematics. Spine J 10:341-349, 2010

5. Denozière G, Ku DN: Biomechanical comparison between fusion of two vertebrae and implantation of an artificial intervertebral disc. J Biomech 39:766-775, 2006

6. Dooris AP, Goel VK, Grosland NM, Gilbertson LG, Wilder DG: Load-sharing between anterior and posterior elements in a lumbar motion segment implanted with an artificial disc. Spine (Phila Pa 1976) 26:E122-E129, 2001

7. Goel VK, Grauer JN, Patel TCh, Biyani A, Sairyo K, Vishnubhotla S, et al: Effects of charité artificial disc on the implanted and adjacent spinal segments mechanics using a hybrid testing protocol. Spine (Phila Pa 1976) 30:2755-2764, 2005

8. Goel VK, Kong W, Han JS, Weinstein JN, Gilbertson LG: A combined finite element and optimization investigation of lumbar spine mechanics with and without muscles. Spine (Phila Pa 1976) 18:1531-1541, 1993

9. Grassmann S, Oxland TR, Gerich U, Nolte LP: Constrained testing conditions affect the axial rotation response of lumbar functional spinal units. Spine (Phila Pa 1976) 23:1155-1162, 1998

10. Grevitt MP, Gardner AD, Spilsbury J, Shackleford IM, Baskerville R, Pursell LM, et al: The Graf stabilisation system: early results in 50 patients. Eur Spine J 4:169-175, 1995

11. Grob D, Benini A, Junge A, Mannion AF: Clinical experience with the Dynesys semirigid fixation system for the lumbar spine: surgical and patient-oriented outcome in 50 cases after an average of 2 years. Spine (Phila Pa 1976) 30:324-331, 2005

12. Haher TR, O'Brien M, Felmly WT, Welin D, Perrier G, Choueka J, et al: Instantaneous axis of rotation as a function of the three columns of the spine. Spine (Phila Pa 1976) 17 (6 Suppl):S149-S154, 1992

13. Ilharreborde B, Shaw MN, Berglund LJ, Zhao KD, Gay RE, An KN: Biomechanical evaluation of posterior lumbar dynamic stabilization: an in vitro comparison between Universal Clamp and Wallis systems. Eur Spine J 20:289-296, 2011

14. James KS, Wenger KH, Schlegel JD, Dunn HK: Biomechanical evaluation of the stability of thoracolumbar burst fractures. Spine (Phila Pa 1976) 19:1731-1740, 1994

15. Kaner T, Sasani M, Oktenoglu T, Ozer AF: Dynamic stabilization of the spine: a new classification system. Turk Neurosurg 20:205-215, 2010

16. Karahalios DG, Kaibara T, Porter RW, Kakarla UK, Reyes PM, Baaj AA, et al: Biomechanics of a lumbar interspinous anchor with anterior lumbar interbody fusion. Laboratory investigation. J Neurosurg Spine 12:372-380, 2010

17. Khoueir P, Kim KA, Wang MY: Classification of posterior dynamic stabilization devices. Neurosurg Focus 22(1):E3, 2007

18. Kikkawa J, Cunningham BW, Shirado O, Hu N, McAfee PC, Oda $\mathrm{H}$ : Biomechanical evaluation of a posterolateral lumbar disc arthroplasty device: an in vitro human cadaveric model. Spine (Phila Pa 1976) 35:1760-1768, 2010

19. Kotani Y, Cunningham BW, Abumi K, Dmitriev AE, Hu N, Ito $\mathrm{M}$, et al: Multidirectional flexibility analysis of anterior and posterior lumbar artificial disc reconstruction: in vitro human cadaveric spine model. Eur Spine J 15:1511-1520, 2006

20. Kumaresan S, Yoganandan N, Pintar FA: Finite element modeling approaches of human cervical spine facet joint capsule. J Biomech 31:371-376, 1998

21. Lu YM, Hutton WC, Gharpuray VM: Do bending, twisting, and diurnal fluid changes in the disc affect the propensity to prolapse? A viscoelastic finite element model. Spine (Phila Pa 1976) 21:2570-2579, 1996

22. Niosi CA, Zhu QA, Wilson DC, Keynan O, Wilson DR, Oxland TR: Biomechanical characterization of the three-dimensional kinematic behaviour of the Dynesys dynamic stabilization system: an in vitro study. Eur Spine J 15:913-922, 2006

23. Panjabi MM, Kato Y, Hoffman H, Cholewicki J, Krag M: A study of stiffness protocol as exemplified by testing of a burst fracture model in sagittal plane. Spine (Phila Pa 1976) 25:2748-2754, 2000

24. Pingel TH: Beitrag zur Herleitung und numerischen Realisierung eines mathematischen Modells der menschlichen Wirbelsäule [dissertation]. Bochum, Germany: Ruhr-Universität Bochum, 1991

25. Pintar FA, Cusick JF, Yoganandan N, Reinartz J, Mahesh M: The biomechanics of lumbar facetectomy under compressionflexion. Spine (Phila Pa 1976) 17:804-810, 1992

26. Polikeit A, Ferguson SJ, Nolte LP, Orr TE: Factors influencing stresses in the lumbar spine after the insertion of intervertebral cages: finite element analysis. Eur Spine J 12:413-420, 2003

27. Rohlmann A, Zander T, Bergmann G: Effect of total disc replacement with ProDisc on intersegmental rotation of the lumbar spine. Spine (Phila Pa 1976) 30:738-743, 2005

28. Rohlmann A, Zander T, Schmidt H, Wilke HJ, Bergmann G: Analysis of the influence of disc degeneration on the mechani- 


\section{Load sharing with respect to COR location}

cal behaviour of a lumbar motion segment using the finite element method. J Biomech 39:2484-2490, 2006

29. Rousseau MA, Cottin P, Levante S, Nogier A, Lazennec JY, Skalli W: In vivo kinematics of two types of ball-and-socket cervical disc replacements in the sagittal plane: cranial versus caudal geometric center. Spine (Phila Pa 1976) 33:E6-E9, 2008 (Erratum in Spine 33:829, 2008)

30. Ruberté LM, Natarajan RN, Andersson GB: Influence of single-level lumbar degenerative disc disease on the behavior of the adjacent segments - a finite element model study. J Biomech 42:341-348, 2009

31. Schilling C, Krüger S, Grupp TM, Duda GN, Blömer W, Rohlmann A: The effect of design parameters of dynamic pedicle screw systems on kinematics and load bearing: an in vitro study. Eur Spine J 20:297-307, 2011

32. Schmidt H, Heuer F, Claes L, Wilke HJ: The relation between the instantaneous center of rotation and facet joint forces - a finite element analysis. Clin Biomech (Bristol, Avon) 23:270 278, 2008

33. Schmidt H, Heuer F, Drumm J, Klezl Z, Claes L, Wilke HJ: Application of a calibration method provides more realistic results for a finite element model of a lumbar spinal segment. Clin Biomech (Bristol, Avon) 22:377-384, 2007

34. Schmidt H, Heuer F, Simon U, Kettler A, Rohlmann A, Claes L, et al: Application of a new calibration method for a threedimensional finite element model of a human lumbar annulus fibrosus. Clin Biomech (Bristol, Avon) 21:337-344, 2006

35. Schmidt H, Heuer F, Wilke HJ: Interaction between finite helical axes and facet joint forces under combined loading. Spine (Phila Pa 1976) 33:2741-2748, 2008

36. Schmidt H, Midderhoff S, Adkins K, Wilke HJ: The effect of different design concepts in lumbar total disc arthroplasty on the range of motion, facet joint forces and instantaneous center of rotation of a L4-5 segment. Eur Spine J 18:1695-1705, 2009

37. Schmoelz W, Onder U, Martin A, von Strempel A: Non-fusion instrumentation of the lumbar spine with a hinged pedicle screw rod system: an in vitro experiment. Eur Spine J 18:1478-1485, 2009

38. Schmoelz W, Schaser KD, Knop C, Blauth M, Disch AC: Extent of corpectomy determines primary stability following isolated anterior reconstruction in a thoracolumbar fracture model. Clin Biomech (Bristol, Avon) 25:16-20, 2010

39. Seligman JV, Gertzbein SD, Tile M, Kapasouri A: Computer analysis of spinal segment motion in degenerative disc disease with and without axial loading. Spine (Phila Pa 1976) 9:566-573, 1984
40. Smit TH, Odgaard A, Schneider E: Structure and function of vertebral trabecular bone. Spine (Phila Pa 1976) 22:28232833, 1997

41. Spitzer V, Ackerman MJ, Scherzinger AL, Whitlock D: The visible human male: a technical report. J Am Med Inform Assoc 3:118-130, 1996

42. Stoll TM, Dubois G, Schwarzenbach O: The dynamic neutralization system for the spine: a multi-center study of a novel nonfusion system. Eur Spine J 11 (Suppl 2):S170-S178, 2002

43. Takigawa T, Espinoza Orías AA, An HS, Gohgi S, Udayakumar RK, Sugisaki K, et al: Spinal kinematics and facet load transmission after total disc replacement. Spine (Phila Pa 1976) 35:E1160-E1166, 2010

44. White AA III, Panjabi MM: Clinical Biomechanics of the Spine, ed 2. Philadelphia: Lippincott Williams \& Wilkins, 1990

45. Xia Q, Wang S, Kozanek M, Passias P, Wood K, Li G: Invivo motion characteristics of lumbar vertebrae in sagittal and transverse planes. J Biomech 43:1905-1909, 2010

46. Zander T, Rohlmann A, Bergmann G: Influence of different artificial disc kinematics on spine biomechanics. Clin Biomech (Bristol, Avon) 24:135-142, 2009

47. Zander T, Rohlmann A, Burra NK, Bergmann G: Effect of a posterior dynamic implant adjacent to a rigid spinal fixator. Clin Biomech (Bristol, Avon) 21:767-774, 2006

48. Zander T, Rohlmann A, Klöckner C, Bergmann G: Influence of graded facetectomy and laminectomy on spinal biomechanics. Eur Spine J 12:427-434, 2003

49. Zhu Q, Larson CR, Sjovold SG, Rosler DM, Keynan O, Wilson DR, et al: Biomechanical evaluation of the Total Facet Arthroplasty System: 3-dimensional kinematics. Spine (Phila Pa 1976) 32:55-62, 2007

Manuscript submitted May 19, 2013.

Accepted January 14, 2014.

Current affiliation for Mr. Alapan: Department of Mechanical \& Aerospace Engineering, Case Western Reserve University, Cleveland, $\mathrm{OH}$.

Please include this information when citing this paper: published online February 28, 2014; DOI: 10.3171/2014.1.SPINE13426.

Address correspondence to: Serkan İnceoğlu, Ph.D., Orthopedic Biomechanics Laboratory, Department of Orthopedic Surgery, Loma Linda University, 11406 Loma Linda Dr., Ste. 218, Loma Linda, CA 92354. email: sinceoglu@1lu.edu. 